\title{
Computational Complexity of Multitape Turing Machines and Random Access Machines
}

\author{
By \\ Takumi KASAI*
}

\section{§1. Introduction}

In recent years there has been an increasing interest in analyzing the computational complexity of programs. The multitape Turing machine has become the standard model used for evaluating time and storage complexity, even though such machines are not much like any existing computers. Some authors, however, implement their algorithms not on Turing machines but on random access machines. In 1972 Cook introduced a formal model of a random access machine. This model is closer to real computer, for real computers calculate the address of desired storage cell within a short time before fetching its content.

Notation. Let $N$ denote the set of natural numbers and let $[k]$ $=\{0,1, \ldots, k-1\}$ for each $k \in N$. Hence $[0]=\varnothing$. We regard [k] as an alphabet consisting of $k$ symbols. Thus, a language is a subset of $[k]^{*}$ for some $k \in N$.

Let $I$ and $O$ be sets. We denote by $[I \rightarrow O]$ the set of all partial functions from $I$ to $O$.

Definition 1.1. A computing machine is a 3-tuple $M=(L, \mathscr{I}, \mathbf{t})$, where

(i) $L$ is a language,

(ii) $\mathscr{I}$ is a function from $L$ to $[I \rightarrow O]$, and

(iii) $\mathbf{t}$ is a function from $L$ to $[I \rightarrow N]$ satisfying the following condition: for each $P \in L$ and $x \in I$,

Received August 10, 1976.

* Research Institute for Mathematical Sciences, Kyoto University, Kyoto 606, Japan. 


$$
\mathscr{I}(P)(x) \text { is defined iff } \mathbf{t}(P)(x) \text { is defined. }
$$

The function $\mathscr{I}$ is called the interpretation of $M$ and $\mathscr{I}(P)$ is the partial function realized by $P$ under $M$. We say that $t(P)$ is the time complexity of $P$, and sometimes write $\mathbf{t}(P, x)$ instead of $t(P)(x)$. The set $I$ is the input domain and $O$ is the output domain.

Definition 1.2. Let $M=(L, \mathscr{I}, \mathfrak{t})$ and $M^{\prime}=\left(L^{\prime}, \mathscr{I}^{\prime}, \mathbf{t}^{\prime}\right)$ be computing machines with the same input domain $I$ and output domain $O$. Let $f: N \rightarrow N$ be a function. Then $M$ is said to be $f(n)$-translatable to $M^{\prime}$ if and only if for each $P \in L$, there exist $P^{\prime} \in L^{\prime}$ and constant $c$ satisfying the following conditions:

$$
\mathscr{I}(P)=\mathscr{I}^{\prime}\left(P^{\prime}\right)
$$

(1.3) for each $x \in I$, if $\mathbf{t}(P, x)$ is defined, then $\mathbf{t}^{\prime}\left(P^{\prime}, x\right) \leqq c f(\mathbf{t}(P, x))$,

that is, if a program $P$ in $L$ takes time $\tau(x)$ for its execution, then there is a program $P^{\prime}$ in $L^{\prime}$ which computes the same partial function as $P$ within time $c f(\tau(x))$. If $f(n)=n$, we say that $M$ is linearly translatable to $M^{\prime}$. If $f$ is a polynomial, then $M$ is polynomially translatable to $M^{\prime}$.

In this paper, we consider the following types of computing machines:

RAM $\cdots$ the random access machine with indirect addressing,

RAMR $\cdots$ the random access machine without indirect addressing,

SM $\quad \cdots \quad$ the step machine with indirect addressing,

SMR $\cdots$ the step machine without indirect addressing,

TM $\quad \cdots \quad$ the on-line multitape Turing machine.

We compare these models on the basis of their ability to reflect the complexity of an algorithm. The results obtained in this paper are summarized in Fig. 7.1. In [7], Cook has shown that the RAM is $n^{2}$-translatable to the TM. In Section 5, we show that this upper bound cannot be improved, that is, we show that the RAM is not $n^{2-\varepsilon_{-}}$ translatable to the TM for any $\varepsilon>0$. This yields a negative answer in the case of on-line model to an open problem suggested by Borodin 
[5] and Aho, Hopcroft and Ullman [2].

One of the purpose of this paper is to construct a good model to use in the theory of computational complexity. We maintain that the SM is a good model, since both RAM and TM (and hence, any restricted type of these machines) are linearly translatable to SM.

\section{§2. Random Access Machine}

Definition 2.1. Let $D$ be the set of functions $d: N \rightarrow N$. Each element $d$ of $D$ is called a memory. For each $i \in N, d(i)$ represents the contents of register $i$. For each $d \in D$ and $i, j \in N$, let $d(i \leftarrow j)$ be the memory defined by

$$
d(i \leftarrow j)(k)=\left\{\begin{array}{lll}
d(k) & \text { if } & k \neq i \\
j & \text { if } \quad k=i .
\end{array}\right.
$$

For each $n \in N$, let

$$
\log n= \begin{cases}\left\ulcorner\log _{2} n\right\urcorner & \text { if } n \geqq 2 \\ 1 & \text { if } n<2 .\end{cases}
$$

Definition 2.2. The RAM instructions, together with their meanings

\begin{tabular}{|c|c|c|}
\hline Instruction & next memory & execution time for RAM \\
\hline 1. $\operatorname{LOAD} n$ & $d(0 \leftarrow d(n))$ & $\log n+\log d(n)$ \\
\hline 2. SETC $n$ & $d(0 \leftarrow n)$ & $\log n$ \\
\hline 3. STORE $n$ & $d(n \leftarrow d(0))$ & $\log n+\log d(0)$ \\
\hline 4. $\operatorname{READ} n$ & $d(0 \leftarrow$ “'input”) & $\log n+\log$ "input" \\
\hline 5. WRITE $n$ & $d$ & $\log n+\log d(n)$ \\
\hline 6. JZERO $n$ & $d$ & $\log n$ \\
\hline 7. $\operatorname{ADD} n$ & $d(0 \leftarrow d(0)+d(n))$ & $\log n+\log d(0)+\log d(n)$ \\
\hline 8. $\operatorname{SUB} n$ & $d(0 \leftarrow d(0)-d(n))$ & $\log n+\log d(0)+\log d(n)$ \\
\hline 9. INCR $n$ & $d(0 \leftarrow d(0)+1)$ & $\log d(0)$ \\
\hline 10. $\mathrm{DECR} n$ & $d(0 \leftarrow d(0)-1)$ & $\log d(0)$ \\
\hline 11. $\mathrm{LOAD} * n$ & $d(0 \leftarrow d(d(n)))$ & $\log n+\log d(n)+\log d(d(n))$ \\
\hline 12. $\mathrm{STORE} * n$ & $d(d(n) \leftarrow d(0))$ & $\log n+\log d(n)+\log d(0)$ \\
\hline
\end{tabular}
TABLE 2.1 RAM Instructions and Execution Times 
and execution times, are given in Table 1.1, where $n$ is an element of $N$ and $d$ represents a current memory.

Definition 2.3. (a) A RAM program is a finite sequence of RAM instructions. (b) A RAMR program is a RAM program without the instruction types $\mathrm{LOAD} * n$ and $\mathrm{STORE} * n$. (c) A SM program is a RAM program with neither ADD nor SUB. (d) A SMR program is a RAM program without ADD, SUB, LOAD $* n$ and STORE $* n$. Thus, it is a $\mathrm{SM}$ program without $\mathrm{LOAD} * n$ and $\mathrm{STOR} * n$.

Definition 2.4. An element $(i, x, y, d)$ of $N \times N^{*} \times N^{*} \times D$ is called a configuration of random access machines. Let $P=s_{1} s_{2} \cdots s_{k}$ be a program with $s_{i}$ being instructions. Let $\left.\right|_{\bar{P}}$ be the relation over the configurations defined as follows. We write

$$
\left.(i, x, y, d)\right|_{\bar{P}}\left(i^{\prime}, x^{\prime}, y^{\prime}, d^{\prime}\right)
$$

if and only if the following conditions are satisfied:

(i) $1 \leqq i \leqq k$,

(ii) if $s_{i}$ is JZERO $n$ and $d(0)=0$ then $i^{\prime}=n$ else $i^{\prime}=i+1$,

(iii) if $s_{i}$ is $\operatorname{READ} n$ then $x=a \cdot x^{\prime}$ for some $a \in N$ else $x^{\prime}=x$

(iv) if $s_{i}$ is WRITE $n$ then $y^{\prime}=y \cdot d(n)$ else $y^{\prime}=y$,

(v) $d^{\prime}$ is the next memory determined by Table 2.1 .

Let $\left.\right|_{P} ^{*}$ be the reflexive transitive closure of $\left.\right|_{\bar{P}}$. If $\left.\alpha\right|_{P} ^{*} \beta$ and there is no $\gamma$ such that $\left.\beta\right|_{\bar{P}} \gamma$, then we write $\left.\alpha\right|_{\hat{P}} \beta$.

Let $d_{0}$ be the memory defined by

$$
d_{0}(i)=0 \quad \text { for all } \quad i \in N \text {. }
$$

Let $\mathscr{I}(P): N^{*} \rightarrow N^{*}$ be the partial function defined by

$$
\mathscr{I}(P)(x)=y \text { iff }\left(1, x, \lambda, d_{0}\right) \hat{\wedge}\left(i, \lambda, y, d^{\prime}\right)
$$

for some $i \in N$ and $d^{\prime} \in D . \mathscr{I}(P)$ is called the partial function realized by $P$.

Definition 2.5. (a) Time complexity of RAM and RAMR: The time complexity of a RAM program (or a RAMR program) $P$ is the 
function $\mathbf{t}_{\mathrm{RAM}}(P): N^{*} \rightarrow N$ such that $\mathrm{t}_{\mathrm{RAM}}(P)(x)$ is the sum of the execution time taken by each instruction executed on input $x$, where the time required by each instruction is shown in Table 2.1 .

(b) Time complexity of SM and SMR: The time complexity of a SM program (or a SMR program) $P$ is the function $\mathbb{t}_{\mathrm{SM}}(P): N^{*} \rightarrow N$ such that $\mathbf{t}_{\mathrm{SM}}(P)(x)$ is the number of instruction steps executed by $P$ on input $x$. That is, in these machine, each instruction requires one unit of time.

Henceforth, the subscript $M$ on $\mathbf{t}_{\mathrm{M}}$ is dropped whenever $M$ is understood.

Definition 2.6. Let $x=x_{1} \cdot x_{2} \cdots x_{n}$ be an element of $N^{*}$ with each $x_{i}$ being in $N$. The proper length of $x$, denoted by $\ln (x)$, is defined by

$$
\ln (x)=\sum_{i=1}^{n} \log x_{i}
$$

Let $f: N \rightarrow N$ be a monotone increasing function and let $P$ be a program. Then $P$ executes within time $f$ (alternatively, $P$ is said to be $f(n)$ time bounded) if and only if

$$
\mathfrak{t}(P, x) \leqq f(\ln (x)) \quad \text { for all } \quad x \in N^{*} .
$$

A language $L \subset[k]^{*}$ is recognized by a program $P$ if $L=\operatorname{Dom} \mathscr{I}(P)$. $L$ is recognizable within time $f$, abbreviated f-recognizable, if there is a program $P$ recognizing $L$ which executes within time $f$.

Definition 2.7. Let $f$ be a partial function from $N^{*}$ to $N^{*}$. Then $f$ is said to be of rank $k$ if

$$
\operatorname{Dom} f \subset[k]^{*} \text { and } \operatorname{Im} f \subset[k]^{*} \text {. }
$$

A program $P$ is said to be of rank $k$ if the partial function realized by $P$ is of rank $k$. In this paper, unless stated otherwise, any program is supposed to be of finite rank.

Remark. Note that any partial function realized by a Turing machine is of finite rank. Now we show that the condition of Definition 
2.7 is not too severe, that is, we show that any RAM program of infinite rank can be simulated within an $n \log n$ factor by a RAM program of finite rank. Let $A=\left(1(0 \cup 1)^{*} 2 \cup 02\right)^{*}$. Let $\xi: N^{*} \rightarrow A$ and $v: A \rightarrow N^{*}$ be the functions defined by

$$
\begin{aligned}
& \xi\left(x_{1} \cdot x_{2} \cdots x_{n}\right)=\bar{x}_{1} 2 \bar{x}_{2} 2 \cdots \bar{x}_{n} 2 \\
& v\left(\bar{x}_{1} 2 \bar{x}_{2} 2 \cdots \bar{x}_{n} 2\right)=x_{1} \cdot x_{2} \cdots x_{n},
\end{aligned}
$$

where $\bar{x}_{i}$ is the binary representation of the integer $x_{i}$.

Then, by the proof of Theorem 4.1 in Section 4, it follows that for any RAM program $P$, there exist a constant $c$ and a $\operatorname{RAM}$ program $\bar{P}$ of rank 3 such that

$$
\begin{aligned}
& \mathscr{I}(P)=v \cdot \mathscr{I}(\bar{P}) \cdot \xi, \quad \text { and } \\
& \mathbb{t}(\bar{P}, \xi(x)) \leqq c \cdot \mathbf{t}(P, x) \cdot \log \mathfrak{t}(P, x) .
\end{aligned}
$$

\section{§3. Relationship between the RAM and the SM}

Theorem 3.1. Let $P$ be a SM program. Then there exists a constant $c$ such that

$$
\mathbf{t}_{\mathrm{RAM}}(P, x) \leqq c \cdot \mathbf{t}_{\mathrm{SM}}(P, x) \log \mathbf{t}_{\mathrm{SM}}(P, x)
$$

Proof. Let $q$ be the largest constant appearing as the argument of SETC instruction in $P$. Let $P$ be of rank $k$. Then, a number appearing in any register during the computation is less than $q+k+\mathbf{t}_{\mathrm{SM}}(P, x)$. Hence one instruction costs at most $O\left(\log t_{\mathrm{SM}}(P, x)\right)$ time under the logarithmic cost criterion.

Corollary 3.1. The $\mathrm{SM}$ is $n \log n$ translatable to the RAM. The $\mathrm{SMR}$ is $n \log n$ translatable to the RAMR.

Notation. Let $L_{0}$ be the language defined by

$$
L_{0}=\left\{w 2 w^{R} 2 \mid w \in\{0,1\}^{*}\right\}
$$

where $w^{R}$ denotes the reversal of word $w$. 
Lemma 3.1. $L_{0}$ is recognizable by a SM program which executes within time $f(n)=c n$ for some constant $c$.

Proof. Evident.

From Theorem 3.1 and Lemma 3.1, we have the following:

Corollary 3.2. $L_{0}$ is recognizable by a RAM program which executes within time $f(n)=c n \log n$ for some constant $c$.

The SMR can be views as a Neuman-type model realization for counter machines $[10,11]$. The following lemma is an immediate consequence of the result obtained by Fischer, Meyer and Rosenberg [11].

Lemma 3.2. If $L_{0}$ is recognizable by a SMR program which executes within time $f(n)$, then $f(n) \geqq c^{n}$ for some constant $c>1$ and for all $n$.

Combining Lemmas 3.1 and 3.2, we have the following result.

Corollary 3.3. The SM is not polynomially translatable to the SMR.

Lemma 3.3. If $L_{0}$ is recognizable by a RAMR program $P$ which executes within time $f(n)$, then $f(n) \geqq c n^{2}$ for some constant $c$ and for all $n$.

Proof. Let $q$ be the largest constant appearing as the argument of a SETC instruction in $P$. First we show that if $m$ is the largest number appearing in any register after a computation of duration $\tau$, then

$$
\tau \geqq \frac{1}{2}\left(\log ^{2} m-\log ^{2} q\right)
$$

The proof will proceed by induction on the length of a computation. It is trivially true for computations of length 0 , since a computation begins with all registers set to zero. Assuming that it is true for a computation 


$$
\left.\left(1, u, \lambda, d_{0}\right)\right|_{P} ^{*}(i, v, \lambda, d)
$$

consider the next move of this computation. We may assume that the $i$ th instruction of $P$ is of the form $\operatorname{ADD} p$. Since

$$
\tau \geqq \frac{1}{2}\left(\log ^{2} \max \{d(0), d(p)\}-\log ^{2} q\right),
$$

it follows that

$$
\begin{aligned}
& \tau+\log d(0)+\log d(p)+\log p \\
& \geqq \frac{1}{2}\left(\log ^{2} \max \{d(0), d(p)\}-\log ^{2} q\right)+\log d(0)+\log d(p) \\
& \geqq \frac{1}{2}\left(\{\log \max \{d(0), d(p)\}+1\}^{2}-\log ^{2} q\right) \\
& \geqq \frac{1}{2}\left(\log ^{2}(d(0)+d(p))-\log ^{2} q\right)
\end{aligned}
$$

Therefore (3.1) holds for all computations.

Let $l$ be the length of $P$ and let $k$ be the number of registers used in $P$. Let $m$ be the largest number appearing in any register after reading a word of length $\frac{n}{2}$. Then, for two distinct binary word $u$ and $v$ of length $\frac{n-1}{2}$, if

$$
\begin{aligned}
& \left.\left(1, u 2 u^{R} 2, d_{0}\right)\right|_{P} ^{*}\left(i, u^{R} 2, \lambda, d\right) \text { and } \\
& \left.\left(1, v 2 u^{R} 2, d_{0}\right)\right|_{P} ^{*}\left(i^{\prime}, u^{R} 2, \lambda, d^{\prime}\right),
\end{aligned}
$$

then either $i \neq i^{\prime}$ or $d \neq d^{\prime}$. Hence we have

$$
l \cdot(m+1)^{k} \geqq 2^{\frac{n}{2}} \cdot
$$

From (3.1) and (3.2), it follows that

$$
\tau \geqq c n^{2}
$$

for some constant $c>0$.

Corollary 3.4. If the $\mathrm{SM}$ is $f(n)$ translatable to the RAMR, then 


$$
\sup _{n \rightarrow \infty} \frac{f(n)}{n^{2}}>0
$$

If the $\mathrm{RAM}$ is $f(n)$ translatable to $\mathrm{RAMR}$, then

$$
\sup _{n \rightarrow \infty} \frac{f(n) \log ^{2} n}{n^{2}}>0
$$

Since the language $L_{0}$ can be recognizable in real time by a Turing machine, we have the following result.

Corollary 3.5. If the $\mathrm{TM}$ is $f(n)$ translatable to RAMR, then

$$
\sup _{n \rightarrow \infty} \frac{f(n)}{n^{2}}>0
$$

\section{§4. Linear Simulation of the RAM by the SM}

In this section, we show that the RAM is linearly translatable to the SM. Since the SM programs to do this are intolerably long, it will be convenient to describe them in a higher-level language called SM-ALGOL, instead of the "machine language" given in Section 2.

Definition 4.1. A SM-ALGOL program call contain one-dimensional infinite arrays.

(a) An atomic statement is one of the followings

$$
\begin{array}{lll}
\text { read } v & \text { write } v & \text { goto label } \\
v \leftarrow w & v \leftarrow w+c & v \leftarrow w-c
\end{array}
$$

where $c$ is a constant and $v$ and $w$ are either simple variables $x$ or subscripted variables of the forms

$$
a[x] \quad a[x+c] \quad a[x-c] .
$$

(b) A condition is one of the followings

$$
v=c \quad v \neq c
$$

where $c$ is a constant and $v$ is a simple variable or a subscripted variable. 
(c) A SM-ALGOL program is a statement of one of the following types.

(1) atomic statement

(2) if condition then statement else statement

(3) if condition then statement

(4) while condition do statement

(5) repeat statement until condition

(6) label: statement

(7) begin statement:..; statement end

(8) procedure name (list of parameters): statement

(9) procedure-name (arguments)

(d) Recursive procedures are not allowed in SM-ALGOL programs, and any procedure statement of type (9) should be previously defined by a procedure declaration of type (8).

The time complexity of a SM-ALGOL program $P$ is the function $\mathbf{t}(P): N^{*} \rightarrow N$ : such that $\mathbf{t}(P)(x)$ is the number of executions of atomic statements and conditions executed by $P$ on input $x$.

Lemma 4.1. Every SM-ALGOL program is linearly translatable to a SM program.

Outline of proof. Let $P$ be a SM-ALGOL program. Without loss of generality we may assume that $P$ contains no procedure call. To prove the lemma, it suffices to show that there exist a SM-ALGOL program $\bar{P}$ with exactly one array and constant $c$ such that

$$
\mathrm{t}(\bar{P}, x) \leqq c t(P, x)
$$

for all inputs $x$.

Let the arrays used in $P$ be $A_{0}, A_{1}, \ldots, A_{k-1}$, and let simple variables used in $P$ be $X_{1}, \ldots, X_{t}$. The program $\bar{P}$ uses a single array $A$ and simple variables $X_{1}, \ldots, X_{t}, X_{1}^{\prime}, \ldots, X_{t}^{\prime}$. The program $\bar{P}$ computes values $v$ and $2 k v$ simultaneously whenever $P$ computes the value $v$, that is, the program $\bar{P}$ can be constructed such that the following relations are satisfied during execution:

$$
X_{i}^{\prime}=2 k \cdot X_{\imath}
$$




$$
\begin{array}{ll}
A[2 k i+j]=A_{j}[i] & 0 \leqq j \leqq k-1 \\
A[2 k i+j+k]=2 k \cdot A_{J}[i] & 0 \leqq j \leqq k-1 .
\end{array}
$$

To do this, for example, the statement $X_{i} \leftarrow X_{j}+c$ in $P$ is translated into

$$
\text { begin } X_{i} \leftarrow X_{j}+c ; \quad X_{i}^{\prime} \leftarrow X_{j}^{\prime}+2 k c \text { end, }
$$

the statement $A_{j}\left[X_{i}\right] \leftarrow X_{t}$ is translated into

$$
\text { begin } A\left[X_{i}^{\prime}+j\right] \leftarrow X_{t} ; \quad A\left[X_{i}^{\prime}+j+k\right] \leftarrow X_{t}^{\prime} \text { end, }
$$

and the statement $X_{t} \leftarrow A_{j}\left[X_{i}\right]$ is translated into

$$
\text { begin } X_{t} \leftarrow A\left[X_{i}^{\prime}+j\right] ; \quad X_{t}^{\prime} \leftarrow A\left[X_{i}^{\prime}+j+k\right] \text { end. }
$$

It should be evident that the program $\bar{P}$ can simulate $P$ faithfully within a constant factor.

Definition 4.2. Let $m$ be a positive integer, and let $m_{0}, m_{1}, \ldots, m_{t}$ be elements of $\{0,1\}$ such that

$$
m_{t}=1, \quad m=\sum_{i=0}^{t} m_{i} 2^{i}
$$

In this paper, the binary representation for $m$ means the word $m_{0} m_{1} \cdots$ $m_{t} 2$. The binary representation for zero is the word consisting a single letter 2 .

Theorem 4.1. The RAM is linearly translatable to the SM.

Outline of proof. Let $P$ be a RAM program. We now construct a SM-ALGOL program $\bar{P}$ which linearly simulates $P$. The program $\bar{P}$ uses arrays ACC, TEMP, INDEX, DATA and CONSm for each constant $m$ appearing as argument of instructions in $P$. Initially, for each constant $m$ appearing in $P$, the binary representation $m_{0} m_{1} \cdots m_{t} 2$ for $m$ is stored in the array CONSm $[0], \ldots, \operatorname{CONSm}[t+1]$.

The array ACC represents the register 0 . The binary representation $a_{0} a_{1} \cdots a_{u+1}$ for the contents $a$ of register $x$ is stored in DATA in a contiguous set of subscripted variables 


$$
\operatorname{DATA}[e]=a_{0}, \quad \operatorname{DATA}[e+1]=a_{1}, \ldots, \operatorname{DATA}[e+u+1]=a_{u+1} \text {. }
$$

The integer $e$ is called the entry corresponding to $x$. If a register $x$ has been used thus far in the computation, then the entry $e$ corresponding to $x$ can be found by means of the array INDEX and the binary representation $x_{0} x_{1} \cdots x_{v+1}$ for $x$, that is, the integers $e_{0} e_{1} \cdots e_{v+1}$ can be found such that

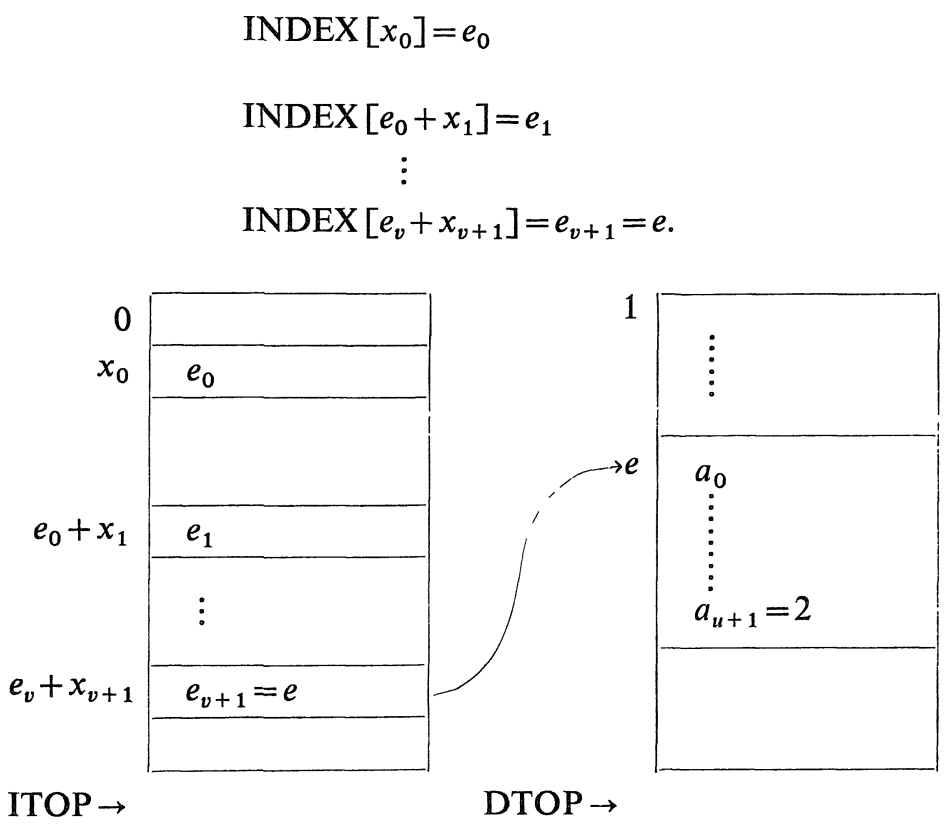

Fig. 4.1.

The procedure $\operatorname{FIND}(X, e)$ finds the entry $e$ corresponding to $X$. The procedure $\operatorname{LOAD}(X, e)$ brings the binary representation $a_{0} a_{1} \cdots a_{u+1}$ to the array $X$. Precisely, these programs are not SM-ALGOL programs, since they contain the statement of the form $e \leftarrow e+X[j]$. This type of statement, however, can be easily translated into a SM-ALGOL program, since $X[j] \leqq 2$ holds whenever this statement is executed. Clearly, the time complexity of $\operatorname{FIND}(X, e)$ is $O(v)$, and hence $O(\log x)$. The time complexity of $\operatorname{LOAD}(X, e)$ is $O(u)$, and hence $O(\log a)$. 


\section{procedure $\operatorname{FIND}(X, e)$ :}

begin

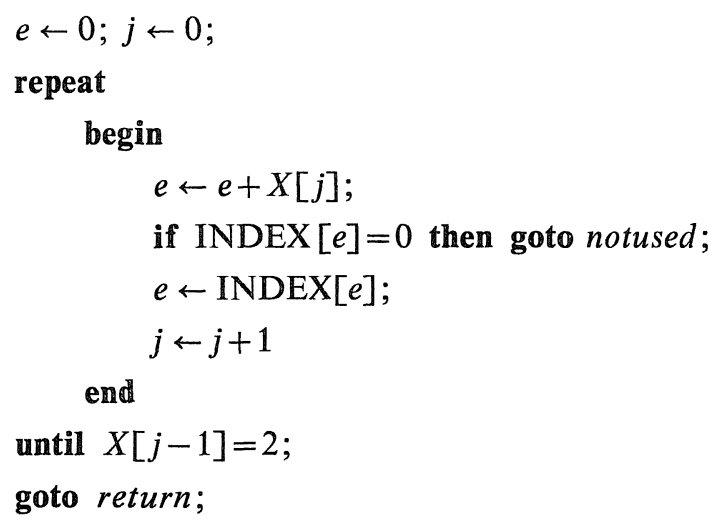

notused: $\quad e \leftarrow 0$;

return:

end

Fig. 4.2. Procedure FIND

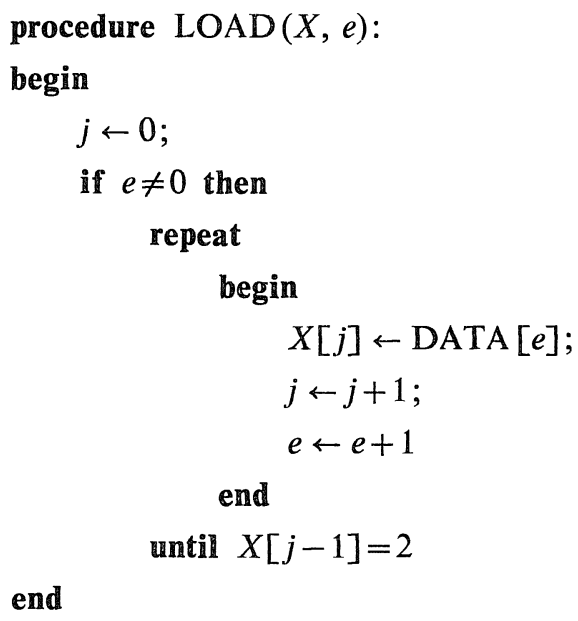

Fig. 4.3. Procedure LOAD

To complete the proof, it suffices to illustrate the simulation of indirect addressing. The statements LOAD $* m$ and STORE $* m$ are simulated by the following SM-ALGOL statements. Now, it should be clear 
that these statements simulate faithfully within a constants factor.

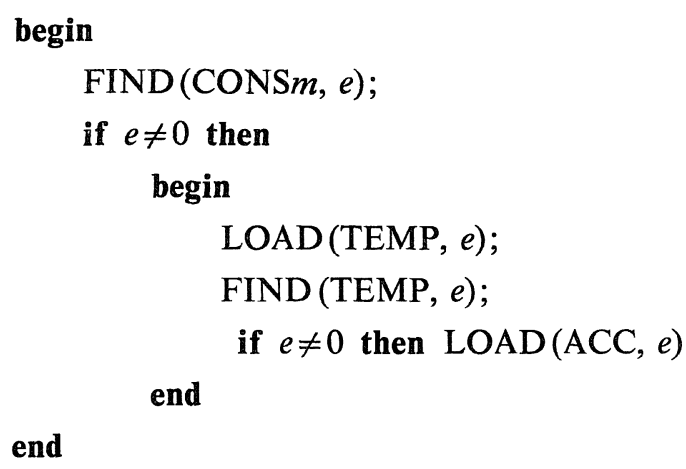

Fig. 4.4. Simulation of LOAD $* m$ by SM

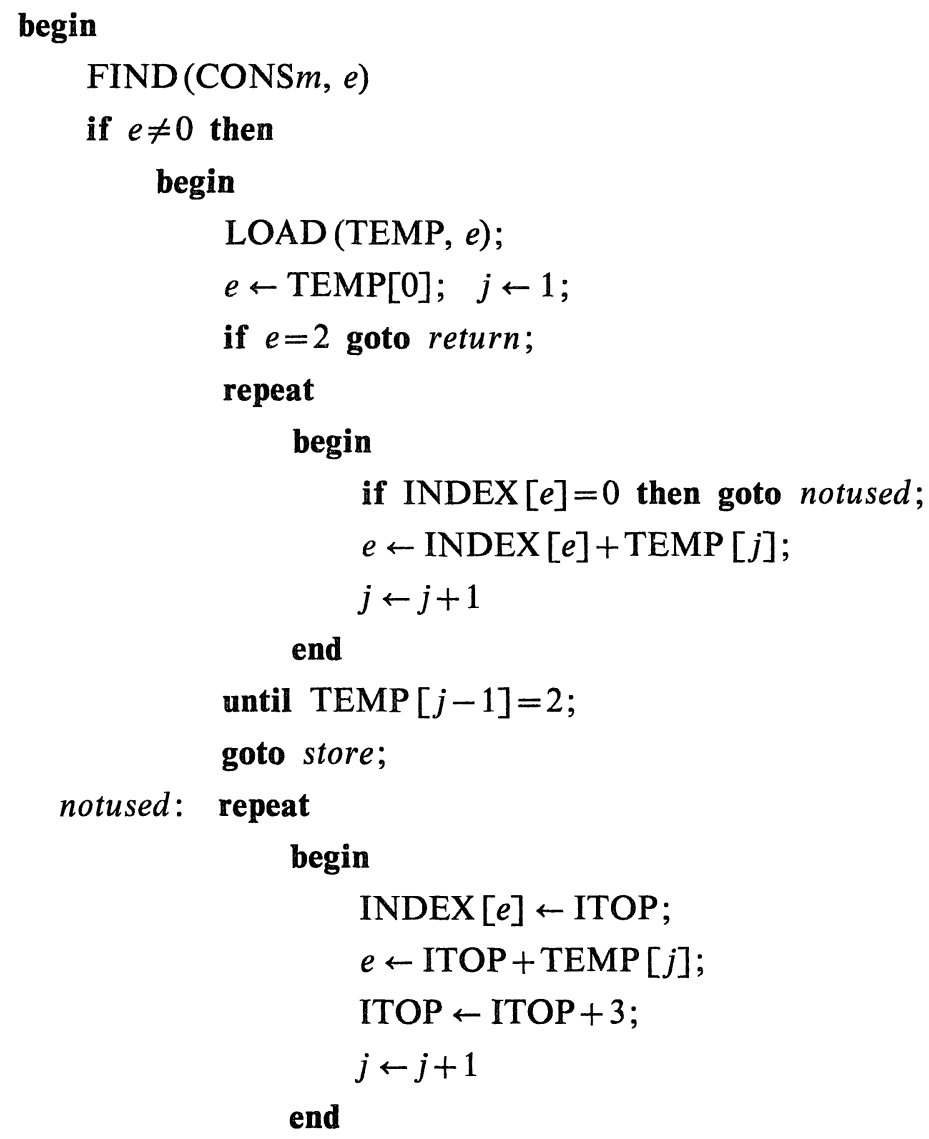


until $X[j-1]=2$;

store: $\quad \operatorname{INDEX}[e] \leftarrow \mathrm{DTOP} ; \quad j \leftarrow 0$;

repeat

begin

$$
\text { DATA }[\mathrm{DTOP}] \leftarrow \mathrm{ACC}[j] ;
$$

$\mathrm{DTOP} \leftarrow \mathrm{DTOP}+1 ; j \leftarrow j+1$

end

until $\mathrm{ACC}[j-1]=2$

end

return:

end

Fig. 4.5. Simulation of STORE $* m$ by SM

\section{§5. Relationship between the TM and the SM}

In this section we show that the SM is not $n^{2-\varepsilon}$ translatable to the TM for any $\varepsilon>0$.

Definition 5.1. Let $U$ be the subset of [4]* defined recursively as follows:

(5.1) $3 \in U$,

(5.2) If $\alpha$ is in $U$, then $0 \alpha$ and $1 \alpha$ are both in $U$,

(5.3) If $\alpha$ and $\beta$ are in $U$, then $2 \alpha \beta$ is in $U$.

For each $\alpha \in U$, let $\varphi(\alpha)$ be the language over $\{0,1\}$ defined as follows:

(5.4) $\varphi(3)=\lambda$,

(5.5) $\varphi(0 \alpha)=0 \varphi(\alpha), \quad \varphi(1 \alpha)=1 \varphi(\alpha)$,

(5.6) $\varphi(2 \alpha \beta)=0 \varphi(\alpha) \cup 1 \varphi(\beta)$,

where $\alpha$ and $\beta$ are elements of $U$.

Lemma 5.1. Let $V$ be any nonempty subset of $\{0,1\}^{i}$. Then there 
exists an element $\alpha$ in $U$ such that

$$
V=\varphi(\alpha) \text { and }|\alpha| \leqq 2^{i+1}-1
$$

Proof. The proof will proceed by induction on $i$. It is trivially true for $i=0$, since $\varphi(3)=\lambda=\{0,1\}^{0}$. Suppose that the lemma is true for all $j<i, i>0$. Let $V_{0}=\{v \mid 0 v \in V\}$ and $V_{1}=\{v \mid 1 v \in V\}$. Then, $V_{k} \subset$ $\{0,1\}^{i-1}$ for $k=0,1$. Thus, by the induction hypothesis there exist $\alpha$ and $\beta$ in $U$ such that

$$
\begin{aligned}
& V_{0}=\varphi(\alpha), \quad V_{1}=\varphi(\beta) \\
& |\alpha| \leqq 2^{i}-1, \quad|\beta| \leqq 2^{i}-1 .
\end{aligned}
$$

Hence

$$
V=0 V_{0} \cup 1 V_{1}=0 \varphi(\alpha) \cup 1 \varphi(\beta)=\varphi(2 \alpha \beta)
$$

and

$$
|2 \alpha \beta|=|\alpha|+|\beta|+1 \leqq 2^{i+1}-1
$$

Therefore the lemma holds for all $i$.

Definition 5.2. Let $L_{1}$ be the language over [5] defined by $L_{1}$ $=U\left(4(0 \cup 1)^{*}\right)^{*} 4$. Let $g:[5]^{*} \rightarrow[2]^{*}$ be the partial function such that

(5.7) $g(y)$ is defined if and only if $y \in L_{1}$,

(5.8) $g\left(\alpha 4 x_{1} 4 \cdots 4 x_{k} 4\right)=b_{1} b_{2} \cdots b_{k}$,

$$
b_{j}=\left\{\begin{array}{lll}
0 & \text { if } & x_{j} \in \varphi(\alpha) \\
1 & \text { if } & x_{j} \notin \varphi(\alpha)
\end{array}\right.
$$

where $\alpha \in U, x_{j} \in[2]^{*}$.

Theorem 5.1. The partial function $g$ can be realized by a SM program in linear time.

Proof. Consider the program MAKETREE in Fig. 5.1. The program MAKETREE terminates if and only if the input $\alpha$ is in $U$. If the program terminates, then the following condition is satisfied at the 
completion of the program execution:

(5.9) a string $b_{0} b_{1} \cdots b_{k}, b_{i} \in\{0,1\}$, is in $\varphi(\alpha)$ if and only if there exist integers $e_{0}, e_{1}, \ldots, e_{k}$ such that

$$
\begin{aligned}
& \operatorname{TREE}\left[2+b_{0}\right]=e_{0} \\
& \operatorname{TREE}\left[e_{0}+b_{1}\right]=e_{1} \\
& \vdots \\
& \operatorname{TREE}\left[e_{k-1}+b_{k}\right]=e_{k} \\
& \operatorname{TREE}\left[e_{k}\right]=1
\end{aligned}
$$

The program MAKETREE uses two stacks TREE and STAK with pointers TRTOP and TOP. It should be clear that the time complexity of MAKETREE is $O(|\alpha|)$. In this program, loop means "dead-end," that is, loop is an abbreviation of while $0=0 \mathrm{do}$.

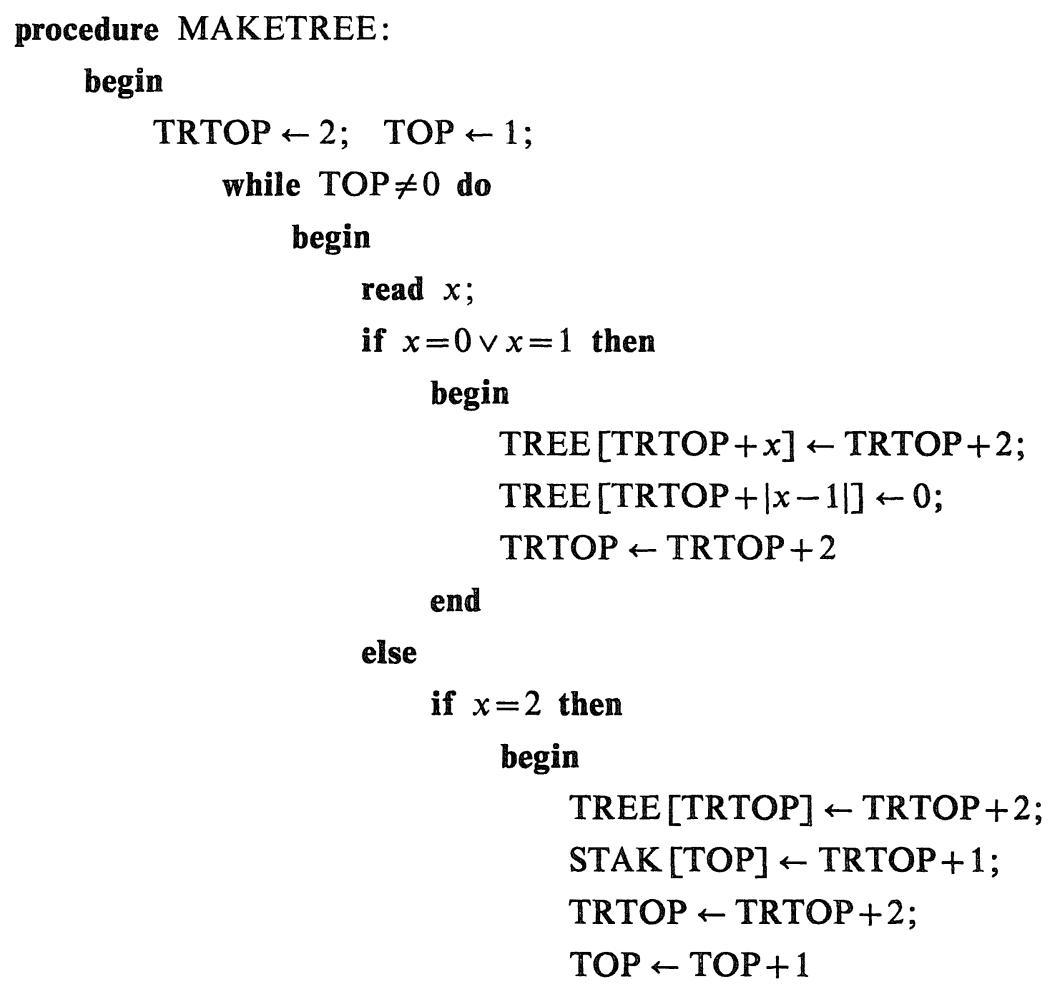






Fig. 5.1. Procedure MAKETREE

The procedure TEST tests whether a given input $x_{j}$ is in $\varphi(\alpha)$ or not, that is, writes 0 on the output tape if $x_{j}$ is in $\varphi(\alpha)$, and writes 1 if $x_{j}$ is not in $\varphi(\alpha)$. The time complexity of TEST is $O\left(\left|x_{j}\right|\right)$. Now it should be clear that the desired function $g$ can be realized by a SM program within time $O(n)$, where $n$ is the length of an input string.

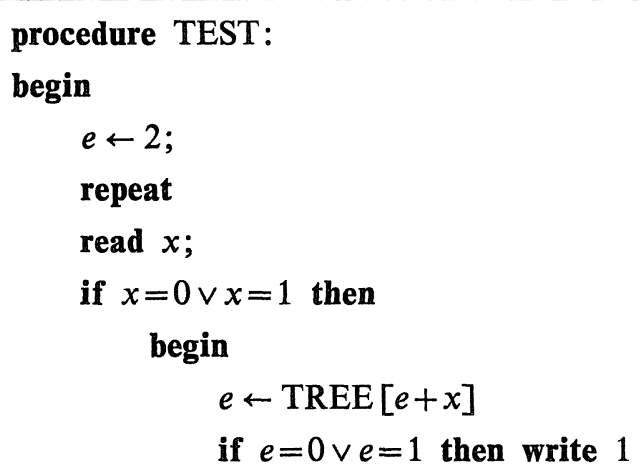




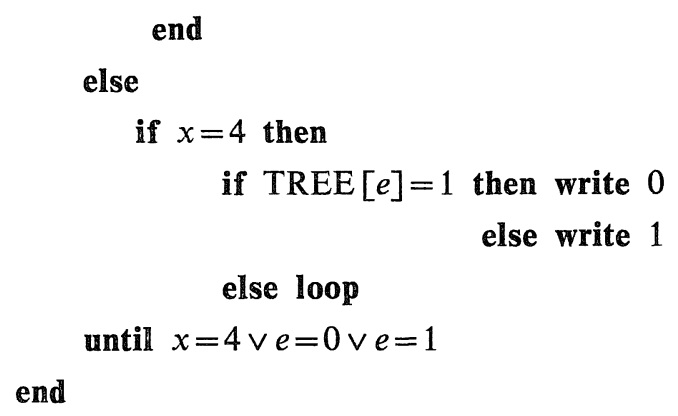

Fig. 5.2. Procedure TEST

Now we show that any Turing machine realizing the partial function $g$ requires at least $n^{2} / \log n$ steps. The Turing machine which we shall use is an ordinary on-line deterministic machine with a one-way read only input tape, a one-way write only output tape and a finite number of two-way, read and write working tapes of unbounded length.

A configuration of a $m$-tape Turing machine $P$ is a 4-tuple

$$
(q, x, y, d),
$$

where $q$ is a state, $x$ is a input tape, $y$ is a output tape and $d \in(N \times$ $\left.N^{*}\right)^{m}$. A pair $(q, d)$ is called tape configuration. We denote by $\left.\right|_{\bar{P}}$ the relation over the configurations which represents one move of the computation of $P$. For each $i \in N$ and configurations $c$ and $c^{\prime}$, we write $c \mid{ }_{P}^{i} c^{\prime}$ if there exists a computation from $c$ to $c^{\prime}$ of length $i$, that is, if there exist configurations $c_{0}, \ldots, c_{i}$ such that

$$
c=\left.\left.\left.c_{0}\right|_{\bar{P}} c_{1}\right|_{\bar{P}} \cdots\right|_{\bar{P}} c_{i}=c^{\prime} .
$$

We write $\left.c\right|_{P} ^{*} c^{\prime}$ iff $\left.c\right|_{P} ^{i} c^{\prime}$ for some $i, c \mid \hat{\vec{P}} c^{\prime}$ iff $\left.c\right|_{\vec{P}} ^{*} c^{\prime}$ and $c^{\prime} \gamma_{\vec{P}} c^{\prime \prime}$ for all $c^{\prime \prime}$, $c \mid \dot{i}^{\wedge} c^{\prime}$ iff $\left.c\right|^{i} c^{\prime}$ and $c \hat{F} c^{\prime}$. The partial function $\mathscr{I}(P): N^{*} \rightarrow N^{*}$ realized by a Turing machine $P$ is defined by

$$
\begin{aligned}
& \mathscr{T}(P)(x)=y \text { iff } \\
& \quad\left(q_{0}, x, \lambda, d_{0}\right) \hat{\bar{P}}\left(q, \lambda, y, d^{\prime}\right) \text { for some } q \text { and } d^{\prime},
\end{aligned}
$$

where $q_{0}$ is the initial state of $P$ and $d_{0}=(0, \lambda)^{m}$.

The time complexity of $P$ is defined by $\mathbf{t}(P)(x)=i$ if and only if 
there exists a configuration $c$ such that

$$
\left.\left(q_{0}, x, \lambda, d_{0}\right)\right|_{\bar{P}} ^{i \wedge} c
$$

Theorem 5.2. If a Turing machine $P$ realizes the partial function $g$ within time $f(n)$, then

$$
f(n) \geqq c \frac{n^{2}}{\log n}
$$

for some $c>0$ and for all $n$.

Proof. Let $P$ be an $m$-tape Turing machine which realizes $g$ within time $f(n)$. Let $A_{i}$ be the subset of $U$ defined by

$$
A_{i}=\left\{\alpha \mid \varphi(\alpha) \subset\{0,1\}^{i}\right\}
$$

By Lemma 5.1,

$$
\# A_{i}=2^{2^{i}}-1 \text {, }
$$

where $\# A$ denotes the number of elements in $A$.

For each $\alpha \in A_{i}$, let $C_{\alpha}$ be the set of tape configurations defined by

$$
\begin{aligned}
C_{\alpha}= & \left\{(q, d) \mid\left(q_{0}, \alpha 4 x_{1} 4 \cdots x_{l} 4, \lambda, d_{0}\right)\right. \\
& \left.\mid \hat{\bar{P}}\left(q, \lambda, b_{1} \cdots b_{l}, d\right), x_{1}, \ldots, x_{l} \in\{0,1\}^{i}\right\}
\end{aligned}
$$

where $q_{0}$ is the initial state of $P$ and $d_{0}=(0, \lambda)^{m}$. Now we show that for $\alpha, \beta \in A_{i}$,

(5.11) if $\alpha \neq \beta$, then $C_{\alpha} \cap C_{\beta}=\varnothing$.

Assume, for contradiction, that $C_{\alpha} \cap C_{\beta} \neq \varnothing$. Let $(q, d) \in C_{\alpha} \cap C_{\beta}$. Then for each $x \in\{0,1\}^{i}$,

$$
x \in \varphi(\alpha)
$$

$$
\begin{aligned}
& \text { iff }(q, x, \lambda, d) \mid \hat{\bar{P}}\left(q^{\prime}, \lambda, 0, d^{\prime}\right) \text { for some } q^{\prime} \text { and } d^{\prime} \\
& \text { iff } x \in \varphi(\beta) .
\end{aligned}
$$

Therefore, $\varphi(\alpha)=\varphi(\beta)$. By definition, it should be clear that $\varphi(\alpha)=\varphi(\beta)$ 
if and only if $\alpha=\beta$. Hence, we have $\alpha=\beta$, contrary to assumption.

Let $P$ have $s$ states and at most $k$ symbols per tape square. We may assume that $k \geqq 2$. Let

$$
h(i)=\frac{2^{i}}{2 m \log k+\log s}-1
$$

Let $F$ be the set of all tape configurations $(q, d)$ which satisfy the following conditions:

(5.13) $(q, d) \in C_{\alpha} \quad$ for some $\quad \alpha \in A_{i}$, and

(5.14) for every $y \in\{0,1\}^{i}$, there exist $t \leqq h(i)$ and a configuration $c$ such that

$$
\left.(q, y, \lambda, d)\right|^{\wedge} c
$$

Next we show that

(5.15) there exists $\alpha \in A_{i}$ such that $C_{\alpha} \cap F=\varnothing$.

Assume, for contradiction, that $C_{\alpha} \cap F \neq \varnothing$ for all $\alpha \in A_{i}$. The only information in storage available to $P$ in next $t$ moves is the present state and the tape information within $t$ squares of the head. From this information, at most $s k^{(2 t+1) m}$ configurations can be distinguished in $t$ moves. Hence, by (5.10) and (5.11) we have

$$
s \cdot k^{(2 h(i)+1) m} \geqq 2^{2^{i}}-1 .
$$

This, however, contradicts (5.12).

Now, consider the following input for $P$ :

$$
z=\alpha 4 x_{1} 4 \cdots x_{l} 4
$$

where $C_{\alpha} \cap F=\varnothing, l=\left[2^{i} / i\right]$ and $x_{1}, \ldots, x_{l}$ are in $\{0,1\}^{i}$. Then, by Lemma 5.1 ,

$$
|z| \leqq 2^{i+1}+\left[2^{i} / i\right] \times i \leqq 2^{i+2}
$$

Consider the following computation:

$$
\left(q_{0}, \alpha 4 x_{1} \cdots 4 x_{l} 4, \lambda, d_{0}\right)
$$




$$
\begin{aligned}
& \mid \frac{t_{0}}{P}\left(q_{1}, x_{1} 4 \cdots x_{l} 4, \lambda, d_{1}\right) \\
& \mid \frac{t_{1}}{P}\left(q_{2}, x_{2} 4 \cdots x_{l} 4, b_{1}, d_{2}\right) \\
& \vdots \\
& \mid \frac{t_{l}}{P}\left(q_{l+1}, \lambda, b_{1} \cdots b_{l}, d_{l+1}\right) .
\end{aligned}
$$

Since $C_{\alpha} \cap F=\emptyset,\left(q_{j}, d_{j}\right)$ is not in $F$ for each $j$. Hence we have

$$
\begin{aligned}
f(|z|) & \geqq t_{1}+\cdots+t_{l} \\
& \geqq h(i)\left[2^{i} / i\right] \\
& \geqq c_{0} 2^{2 i} / i
\end{aligned}
$$

for some constant $c_{0}$ and for all $i$. Hence

$$
f(|z|) \geqq c_{1}|z|^{2} / \log |z|
$$

for some $c_{1}$ and for all $z$. Since $f(n)$ is monotone increasing with $n$, we get

$$
f(n) \geqq c_{1} n^{2} / \log n \text {. }
$$

Corollary 5.1. If the $\mathrm{SM}$ is $f(n)$ translatable to the TM then

$$
\sup _{n \rightarrow \infty} \frac{f(n) \log n}{n^{2}}>0
$$

Combining Corollary 3.1 and Corollary 5.1, we have the following result.

Corollary 5.2. If the RAM is $f(n)$ translatable to the TM then

$$
\sup _{n \rightarrow \infty} \frac{f(n) \log ^{3} n}{n^{2}}>0
$$

Remark. Since it is proved by Cook and Reckhow that the RAM is $n^{2}$ translatable to the TM we can assert that this bound is close to best. 


\section{§6. Simulation of the TM by the RAMR}

In this section we show that the TM is $n^{2}$ translatable to the RAMR.

Definition 6.1. The tape complexity of a Turing machine $P$ is the function $\mathbf{s}_{\mathrm{TM}}(P): N^{*} \rightarrow N$ such that $\mathbf{s}_{\mathrm{TM}}(P)(x)$ is the number of tape squares used in the computation on input $x$.

Definition 6.2. A multi-pushdown tape machine is a Turing machine with a read only input tape, a write-only output tape and a finite number of storage tapes with two storage tape symbols 0 (blank) and 1 . Whenever a head moves left on any one of its storage tape, a "blank" is printed of that tape. Thus, each multi-pushdown tape machine can be viewed as a finite sequence of the following statements (we call this a MPDM program):

\section{(i ) $\operatorname{PUSH} b[i]$}

(ii) $\mathrm{POP}[i]$

(iii) IF TOP $[i]=b$ THEN GOTO $n$

(iv) JF INPUT $=c$ THEN GOTO $n$

\section{(v) WRITE $c$}

where $i, n, c \in N$ and $b \in\{0,1\}$.

The effect of most of the instructions should be evident. For example, $\operatorname{PUSH} b[i]$ causes to print the symbol $b$ on top of the stack $i$. The instruction POP $[i]$ causes to remove the top symbol of the stack $i$, that is, a " 0 " is printed on the tape cell scanned and then the head is moved left one cell.

Lemma 6,1. Let $P$ be a Turing machine. Then there exists a multi-pushdown tape machine (a MPDM program) $\widetilde{P}$ such that

$$
\mathscr{I}(P)=\mathscr{I}(\bar{P})
$$




$$
\begin{array}{r}
\mathbf{t}_{\mathrm{TM}}(\bar{P}, x) \leqq c \mathbf{t}_{\mathrm{TM}}(P, x) \\
\mathbf{s}_{\mathrm{TM}}(\bar{P}, x) \leqq c \mathbf{s}_{\mathrm{TM}}(P, x)
\end{array}
$$

for some constant $c$ and for all $x$.

Proof. Evident.

Definition 6.3. Let top: $[2]^{*} \rightarrow\{0,1, \lambda\}$, pop: $[2]^{*} \rightarrow[2]^{*}$, push0: [2]* $\rightarrow[2]^{*}$, push $1:[2]^{*} \rightarrow[2]^{*}$ be functions defined as follows:

$$
\begin{gathered}
\operatorname{top}(w)=\left\{\begin{array}{lll}
b & \text { if } \quad w=v b, & b \in[2], v \in[2]^{*} \\
\lambda & \text { if } \quad w=\lambda,
\end{array}\right. \\
\operatorname{pop}(w)=\left\{\begin{array}{lll}
v & \text { if } \quad w=v b, \\
\lambda & \text { if } \quad w=\lambda,
\end{array}\right. \\
\quad \operatorname{push} 0(w)=w 0 \\
\operatorname{push} 1(w)=w 1
\end{gathered}
$$

Definition 6.4. For each $w \in[2]^{*}$, let $x_{w}$ and $y_{w}$ be the integers defined recursively as follows:

(i) $x_{\lambda}=0, y_{\lambda}=1$

(ii) if $w=v 0$ then

$$
\begin{aligned}
& x_{w}=x_{v}+2 y_{v} \\
& y_{w}=x_{v}+y_{v}
\end{aligned}
$$

(iii) if $w=v 1$ then

$$
\begin{aligned}
& x_{w}=x_{v}+y_{v} \\
& y_{w}=x_{v}+2 y_{v} .
\end{aligned}
$$

The following results are immediate consequences of the above definition.

Lemma 6.2. For each $w \in[2]^{*}$, 


$$
\begin{aligned}
& x_{w}>y_{w} \quad \text { iff } \quad \text { top }[w]=0 \\
& w_{w}=0 \quad \text { iff } \quad w=\lambda \\
& 0<x_{w}<y_{w} \quad \text { iff } \quad \text { top }[w]=1 \text {. }
\end{aligned}
$$

Lemma 6.3. If $w=v b$ with $b \in[2]$ and $v \in[2]^{*}$, then

$$
\begin{aligned}
& x_{v}=\text { if } x_{w}>y_{w} \text { then } 2 y_{w}-x_{w} \text { else } 2 x_{w}-y_{w} \\
& y_{v}=\text { if } x_{w}>y_{w} \text { then } x_{w}-y_{w} \text { else } y_{w}-x_{w}
\end{aligned}
$$

Lemma 6.4. For every $w \in[2]^{*}$,

$$
x_{w} \leqq 3^{|w|}, \quad y_{w} \leqq 3^{|w|},
$$

Theorem 6.1. For any Turing machine $P$, there exists a RAMR program $\bar{P}$ such that

$$
\begin{aligned}
& \mathscr{I}(P)=\mathscr{I}(\bar{P}) \\
& \mathbf{t}_{\mathrm{RAM}}(\bar{P}, x) \leqq c \mathbf{S}_{\mathrm{TM}}(P, x) \mathbf{S}_{\mathrm{TM}}(P, x)
\end{aligned}
$$

for some constant $c$ and for all $x$.

Proof. By Lemma 6.1, we may assume that $P$ is a MPDM. Let $P$ have $m$ stacks. If the contents of $i$ th stack is $w$, then the integers $x_{w}$ and $y_{w}$ are stored in registers $2 i+1$ and $2 i+2$. Let $X_{i}$ denote the contents of register $i$. The simulation of $P$ proceeds as follows:

(i) PUSH $0[i]$ is simulated by

$$
\begin{aligned}
& X_{2 i+1} \leftarrow X_{2 i+1}+2 X_{2 i+2} \\
& X_{2 i+2} \leftarrow X_{2 i+1}+X_{2 i+2}
\end{aligned}
$$

(ii) PUSH1 [i] is simulated by

$$
\begin{aligned}
& X_{2 i+1} \leftarrow X_{2 i+1}+X_{2 i+2} \\
& X_{2 i+2} \leftarrow X_{2 i+1}+2 X_{2 i+2}
\end{aligned}
$$

(iii) $\mathrm{POP}[i]$ is simulated by 


$$
\begin{array}{r}
X_{2 i+1} \leftarrow \text { if } X_{2 i+1}>X_{2 i+2} \text { then } 2 X_{2 i+2}-X_{2 i+1} \\
\text { else } 2 X_{2 i+2}-X_{2 i+1} \\
X_{2 i+2} \leftarrow \text { if } X_{2 i+1}>X_{2 i+2} \text { then } X_{2 i+1}-X_{2 i+2} \\
\text { else } X_{2 i+2}-X_{2 i+1}
\end{array}
$$

(iv) the condition TOP $[i]$ is simulated by

$$
X_{2 i+1}>X_{2 i+2}
$$

By Lemmas 6.2 and 6.3, it should be clear that the simulations above work correctly. By Lemma 6.4, each simulation requires at most $O(\mathbf{s}(P$, $x)$ ) time. Hence the total time spend by $\bar{P}$ is

$$
O\left(\mathbf{t}_{\mathrm{TM}}(P, x) \cdot \mathbf{s}_{\mathrm{TM}}(P, x)\right) \text {. }
$$

Corollary 6.1. The TM is $n^{2}$ translatable to the RAMR.

Proof. The proof follows from the fact that

$$
\mathbf{s}_{\mathrm{TM}}(P, x) \leqq \mathbf{t}_{\mathrm{TM}}(P, x) .
$$

In [7], Cook and Reckhow show that for each RAM program $P$, there exist a Turing mach $\bar{P}$ and a constant $c>0$ such that

$$
\begin{aligned}
& \mathscr{I}(P)=\mathscr{I}(\bar{P}) \\
& \mathbb{t}_{\mathrm{TM}}(\bar{P}, x) \leqq c \cdot \mathbf{t}_{\mathrm{RAM}}^{2}(P, x) \\
& \mathbf{s}_{\mathrm{TM}}(\bar{P}, x) \leqq c \cdot \mathbb{t}_{\mathrm{RAM}}(P, x) .
\end{aligned}
$$

From this fact, we have the following result.

Corollary 6.2. The RAM is $n^{3}$ translatable to the RAMR.

\section{§7. Conclusion}

In this section, we summarize the results obtained in this paper.

Notation. Let $M$ and $M^{\prime}$ be computing machines. We write (i) 
$M \stackrel{k}{\longrightarrow} M^{\prime}$ if and only if $M$ is $n^{k+\varepsilon}$ translatable to $M^{\prime}$ for any $\varepsilon>0$, but not $n^{k-\varepsilon}$ translatable to $M^{\prime}$ for any $\varepsilon>0$, (ii) $M \stackrel{(1)}{\longrightarrow} M^{\prime}$ if and only if $M$ is linearly translatable to $M^{\prime}$ (iii) $M \stackrel{(2,3)}{\longrightarrow} M^{\prime}$ if and only if $M$ is $n^{3+\varepsilon}$ translatable to $M^{\prime}$ but not $n^{2-\varepsilon}$ translatable to $M^{\prime}$ for any $\varepsilon>0$, (iv) $M \stackrel{\infty}{\longrightarrow} M^{\prime}$ if and only if $M$ is not polynomially translatable to $M^{\prime}$.

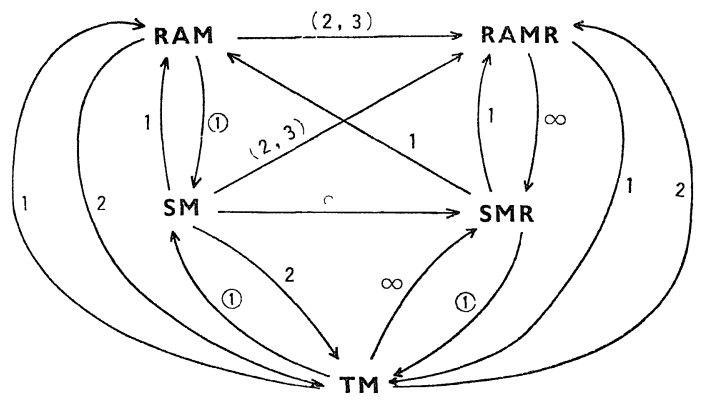

Fig. 7.1

Remark. Since the gap between $n^{k+\varepsilon}$ and $n^{h-\varepsilon}$ is small, the relation $\stackrel{k}{\longrightarrow}$ is practically optimal. However the gap between $n^{2}$ and $n^{3}$ is still wide, and the relation $\stackrel{(2,3)}{\longrightarrow}$ must be improved.

Open problem. Can the upper bound $O\left(n^{3}\right)$ or the lower bound $O\left(n^{2}\right)$ on the time for the RAMR to simulate the RAM be improved?

\section{Acknowledgements}

The author wishes to express his gratitude to Professor Satoru Takasu for his advice. The author is also indebted to Professor Shigeru Igarashi and Mr. Takeshi Hayashi for their suggestions toward this paper.

\section{References}

[1] Aho, A. V., Hopcroft, J. E., and Ullman, J. D., Time and tape complexity of pushdown automaton languages. Information and Control, 13 (1968), 186-206.

[2] Aho, A. V., Hopcroft, J. E., and Ullman, J. D., The design and analysis of computer algorithms, Addison-Wesley, 1974.

[3] Book, R. V., On languages accepted in polynomial time, SIAM J. Comput., 1 (1972), 281-287.

[4] Book, R. V., Greibach, S. A., and Wegbreit, B., Time- and tape-bounded Tur- 
ing accepters and AFL's, J. Comput. System Sci., 4 (1970), 606-621.

[5] Borodin, A., Computational complexity: theory and practice. In "Currents in the theory of computing" (Aho, ed.). Prentice-Hall, Englewood Cliffs, N. J., 1973.

[6] Cook, S. A., Linear time simulation of deterministic two-way pushdown automata, Proc. IFIP Congress 71, TA-2. North-Holland, Amsterdam, (1971), 174179.

[7] Cook, S. A., and Reckhow, R., Time-bounded random access machines, J. Comput. System Sci., 7 (1973), 354-375.

[8] Fischer, P. C., Predecessor Machines, J. Comput. System Sci., 8 (1974), 190-219.

[9] Miller, R. E., and Thatcher, J. W. (eds.), Complexity of Computer Computations, Plenum Press.

[10] Minsky, M., Computation: Finite and Infinite Machines, Prentice-Hall, Englewood Cliffs, N. J., 1967.

[11] Fischer, P. C., Meyer, A. R. and Rosenberg, A. L., Counter machines and counter languages, Math. Systems Theory, 2 (1968), 265-283. 